\title{
Mitochondrial dysfunction driven by the LRRK2-mediated pathway is associated with loss of Purkinje cells and motor coordination deficits in diabetic rat model
}

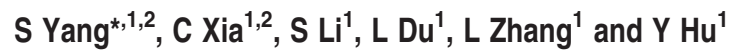

Diabetic neuropathy develops on a background of hyperglycemia and an entangled metabolic imbalance. There is increasing evidence of central nervous system involvement in diabetic neuropathy and no satisfactory treatment except maintenance of good glycemic control, thereby highlighting the importance of identifying novel therapeutic targets. Purkinje cells are a class of metabolically specialized active neurons, and degeneration of Purkinje cells is a common feature of inherited ataxias in humans and mice. However, whether Purkinje cells are implicated in diabetic neuropathy development under metabolic stress remains poorly defined. Here, we revealed a novel leucine-rich repeat kinase 2 (LRRK2)-mediated pathway in Purkinje cells that is involved in the pathogenesis of diabetic neuropathy from a 24-week long study of streptozotocin (STZ)-diabetic rats. We found that hyperglycemia, cerebellum proinflammatory cytokines, and chemokines increased markedly in 24-week STZ-diabetic rats. Furthermore, we demonstrated that degeneration of Purkinje cells is characterized by progressive swellings of axon terminals, no autophagosome formation, the reduction of LC3II/LC3I and Lamp2, and accumulation of p62 puncta in 24-week STZ-diabetic rats. Importantly, a higher expression level of LRRK2-mediated hyperphosphorylation of tau along with increased mitochondrial dynamin-like protein (mito-DLP1) was demonstrated in 24-week STZ-diabetic rats. This effect of LRRK2 overexpression induced mitochondrial fragmentation, and reduced mitochondrial protein degradation rates were confirmed in vitro. As a consequence, 24-week STZ-diabetic rats showed mitochondrial dysfunction in cerebellar Purkinje neurons and coordinated motor deficits evaluated by rotarod test. Our findings are to our knowledge the first to suggest that the LRRK2-mediated pathway induces mitochondrial dysfunction and loss of cerebellar Purkinje neurons and, subsequently, may be associated with motor coordination deficits in STZ-diabetic rats. These data may indicate a novel cellular therapeutic target for diabetic neuropathy.

Cell Death and Disease (2014) 5, e1217; doi:10.1038/cddis.2014.184; published online 8 May 2014

Subject Category: Immunity

Diabetes mellitus is the most common cause of neuropathy and is becoming an increasing burden for both developed and developing countries. ${ }^{1}$ Typical symptoms of diabetic neuropathy include pain, numbness, tingling, weakness, and difficulties with balance. ${ }^{2}$ Multiple biochemical features are implicated in its pathogenesis pointing to systemic and cellular imbalances in metabolites of glucose and lipids. Hyperglycemia results in oxidative stress and elevation of mitochondrial production of reactive oxygen species (ROS) ${ }^{3}$ hypoxia and ischemia lead to an increase in advanced glycation end products, ${ }^{4,5}$ protein kinase $\mathrm{C}$ and $\mathrm{NF}-\kappa \mathrm{B}$, and polyol pathway activation. ${ }^{6,7}$ It is also associated with growth factor deficiency $^{8,9}$ and more complex associated with inflammation at the systemic and cellular level. ${ }^{10-12}$ Although diabetic neuropathy has been long considered to be a disease of the peripheral nervous system, there is now increasing evidence to show that the insult of diabetes may occur at all levels of the nervous system as brain micro/macrovascular disease appears to be associated with cognitive decline and brain atrophy. ${ }^{13}$ Recent advances in neuroimaging methods have led to a better understanding and refinement of how diabetic neuropathy affects the central nervous system (CNS) and to a critical rethinking in a new direction for further research on this disorder. ${ }^{14}$ However, how the CNS responds to metabolic stress is far from clear.

Leucine-rich repeat kinase 2 (LRRK2) is expressed widely in the normal brain, with strong expression in the substantia nigra, basal ganglia, cortex, hippocampus, and cerebellum. ${ }^{15}$ LRRK2 has an important role in cell homeostasis of transmitting signals or helping to assemble the cell's structural framework,

\footnotetext{
${ }^{1}$ Jiangsu Key Laboratory for Molecular and Medical Biotechnology, College of Life Science, Nanjing Normal University, Nanjing, Jiangsu Province 210046, China ${ }^{*}$ Corresponding author: S Yang, Jiangsu Key Laboratory for Molecular and Medical Biotechnology, College of Life Science, Nanjing Normal University, \#1 Wenyuan Road, Yadong District, Nanjing, Jiangsu Province 210046, China. Tel: +86 25 85891242; Fax: +86 25 85891242; E-mail: 08275@njnu.edu.cn

${ }^{2}$ These authors contributed equally to this work.

Keywords: Purkinje cell; motor coordination deficit; mitochondria; LRRK2; streptozotocin-diabetic rat model

Abbreviations: LRRK2, leucine-rich repeat kinase 2; DCN, deep cerebellar nuclei; CNS, central nervous system; MCP-1, monocyte chemotactic protein-1; T2D, type 2 diabetes; ROS, reactive oxygen species; STZ, streptozotocin; Lamp, lysosome-associated membrane protein; LC3I/II, light chain 3I/II; DLP1, dynamin-like protein 1; JC-1, 5,5',6,6'-tetrachloro-1, $1^{\prime}, 3,3^{\prime}$-tetraethylbenzimidazolylcarbocyanine iodide

Received 03.1.14; revised 19.3.14; accepted 24.3.14; Edited by A Stephanou
} 
and turning on and off many cell activities. ${ }^{16}$ Most individuals with LRRK2 mutations have dopamine neuron loss in substantia nigra and Lewy body pathology, suggesting that LRRK2 may have an important role across the spectrum of neurodegenerative disease. ${ }^{3}$ Recent advances in LRRK2 biology have revealed complex multifunctional roles, interacting proteins, and putative physiological substrates in multiple cellular compartments. Through a calcium-dependent pathway, LRRK2 regulates autophagic activities. ${ }^{17,18}$ Too much LRRK2 activity decreases the ratio of light chain 3II (LC3II)/ LC3I, indicators of autophagy, and reduces the level of lysosome-associated membrane protein 2 (LAMP2), a protein critical for conversion of early autophagic vacuoles to vacuoles which rapidly degrade their content, and increases p62 levels by blocking lysosomal degradation of autophagosomes. ${ }^{17}$ In addition, through unclear mechanisms, LRRK2 kinase regulates cytoskeleton architecture through control of protein translation, phosphorylation of cytoskeletal proteins, and response to cellular stressors, ${ }^{19}$ and may be involved upstream in the diabetic neuropathy disease-related chain of events. $^{20}$

Schwann cells and vasa nervorum are the two primary cell types targeted in the pathogenesis of the diabetic neuropathy deregulated pathways and have been intensively studied. ${ }^{21,22}$ Purkinje cells are a class of specialized neurons susceptible to metabolic stress in the cerebellum, as they receive immense synaptic stimulation, and provide the only efferent output from the cerebellum. Purkinje cells can be harmed by a variety of causes: metabolic stress, ${ }^{23}$ autoimmune diseases, ${ }^{24}$ autism, ${ }^{25}$ inherited ataxias, ${ }^{26}$ autophagy deficiency, ${ }^{27,28}$ and neurodegenerative diseases. ${ }^{29,30}$ Adult Purkinje cells' fusion with bone marrow-derived cells in the cerebellum specifically induced by diabetes leads to a subpopulation of highly proinflammatory properties. They seem to have a central role in the pathogenesis of diabetic neuropathy. ${ }^{31,32}$ Thus, the importance of Purkinje cells in diabetic neuropathy is of interest. We surveyed Purkinje cells in the cerebellum of 24-week streptozotocin (STZ)-diabetic rats. These rats extended the measurement period to 24 weeks, which was very important to study the mechanisms of the chronic complications of type 2 diabetes (T2D), such as neuropathy. ${ }^{33}$ We confirmed that the STZ-diabetic rats coexpressed TNF- $\alpha$, IL-6, and monocyte chemotactic protein-1 (MCP-1) in the cerebellum and also demonstrated that STZ-diabetic rats had motor coordination deficits. In addition, we found unexpectedly impaired autophagic activity and loss of Purkinje cells in these diabetic rats. ${ }^{34}$ Further, the effects of higher expression level of LRRK2-mediated hyperphosphorylation of tau along with increased dynamin-like protein 1 (DLP1) were revealed in these diabetic rats. Next, the effects of LRRK2 overexpression on the mitochondrial fragmentation and protein degradation rates were detected in vitro. Finally, we conducted experiments to test mitochondrial function using these rats. Combined together, we identified that the dysfunctional mitochondria and loss of cerebellar Purkinje neurons induced by the diabetic state were as much the perpetrators as these other cell types in diabetic neuropathy. These results may reveal a sequence of chain events that represente a new paradigm for the pathogenesis of diabetic neuropathy in T2D.

\section{Results}

General characterization of STZ-diabetic rats. To assess the effect of diabetes on food and water intake, body weight, blood glucose, and motor coordination function in STZdiabetic rats, we characterized the STZ-diabetic rats every 4 weeks. The food and water intake, body weight, and blood glucose changes were maintained for the 24 weeks of observation. Diabetic rats showed significantly higher intake of food and water when compared with the control group ${ }^{* \star} P<0.01$; Figures $1 \mathrm{a}$ and $\mathrm{b}$ ). The blood glucose concentrations of the STZ-diabetic rats were significantly increased from 4 to 24 weeks $\left({ }^{* *} P<0.01\right.$; Figure $1 \mathrm{~d}$ ), whereas body weights decreased markedly from 4 to 24 weeks ( ${ }^{*} P<0.05$, ${ }^{* \star} P<0.01$; Figure 1c) compared with control rats (nondiabetic rats). The STZ-diabetic rats were given moderate insulin therapy to prevent ketoacidosis without normalizing hyperglycemia. As in humans, only $60 \%$ of STZ-diabetic rats survived the 24-week duration of diabetes. To investigate motor coordination function, we conducted the rotarod test with these diabetic and control rats. It was noticed that, in this test, 24-week STZ-diabetic rats ( $n=8$ /group) exhibited increasing motor deficiencies and fell off the rod more quickly than control rats ( $n=9 /$ group; ${ }^{\star} P<0.05$; Figure $1 \mathrm{e}$ ).

Increased expression levels of proinflammatory cytokine and chemokine in the cerebella of 24-week STZ-diabetic rats. To assess the effect of diabetes on the cerebellum, TNF- $\alpha$, IL- 6 , and MCP- 1 concentrations in STZ-diabetic rats were detected. Protein expression levels of TNF- $\alpha$, IL-6, and MCP-1 were significantly increased at 24 weeks in the cerebella of diabetic rats ( $n=6 /$ group) compared with those of control rats ( $n=6 /$ group) $\left({ }^{*} P<0.05\right.$; Figure 2$)$. The protein expression levels of TNF- $\alpha$, IL-6, and MCP-1 were significantly increased at 24 weeks in the cerebella of diabetic rats compared with the 4-week rats $(\# P<0.05)$, although there was no difference between 4-week diabetic and control rats. These results suggest that chemokines and cytokines take part together in the pathogenesis and progression of T2D neuropathy in 24-week STZ-diabetic rats.

Impaired autophagic activity and accumulated levels of p62 in axon terminal swellings of Purkinje cells in 24-week STZ-diabetic rats. Progressive dysfunction of autophagy could trigger apoptotic cascades and degeneration in Purkinje cells. ${ }^{35}$ To investigate the effects of diabetes on autophagy function, we assayed autophagic activity inside Purkinje cells in 24-week STZ-diabetic rats. We identified autophagic activity impairments and p62 accumulation in Purkinje cells of 24-week STZ-diabetic rats. The failure in activation of the autophagy pathway in 24-week STZ-diabetic rats was reflected by the significant reduction of LC3II/LC3I $\left({ }^{* \star} P<0.01\right)$ and Lamp2 ( $\left.P<0.05\right)$ levels, and elevation of p62 $\left({ }^{\star} P<0.05\right)$ compared with 24 -week control rats (Figure $3 a$ ). The LC3II level was significantly lower than that in 4-week diabetic rats $(\# P<0.05)$. Four-week STZ-diabetic and control rats also exhibited induction of autophagy examined by the above expression pattern of autophagic activity protein (Figure 3a). Purkinje cells failed to display fluorescent puncta, the abolishment of autophagosomes 

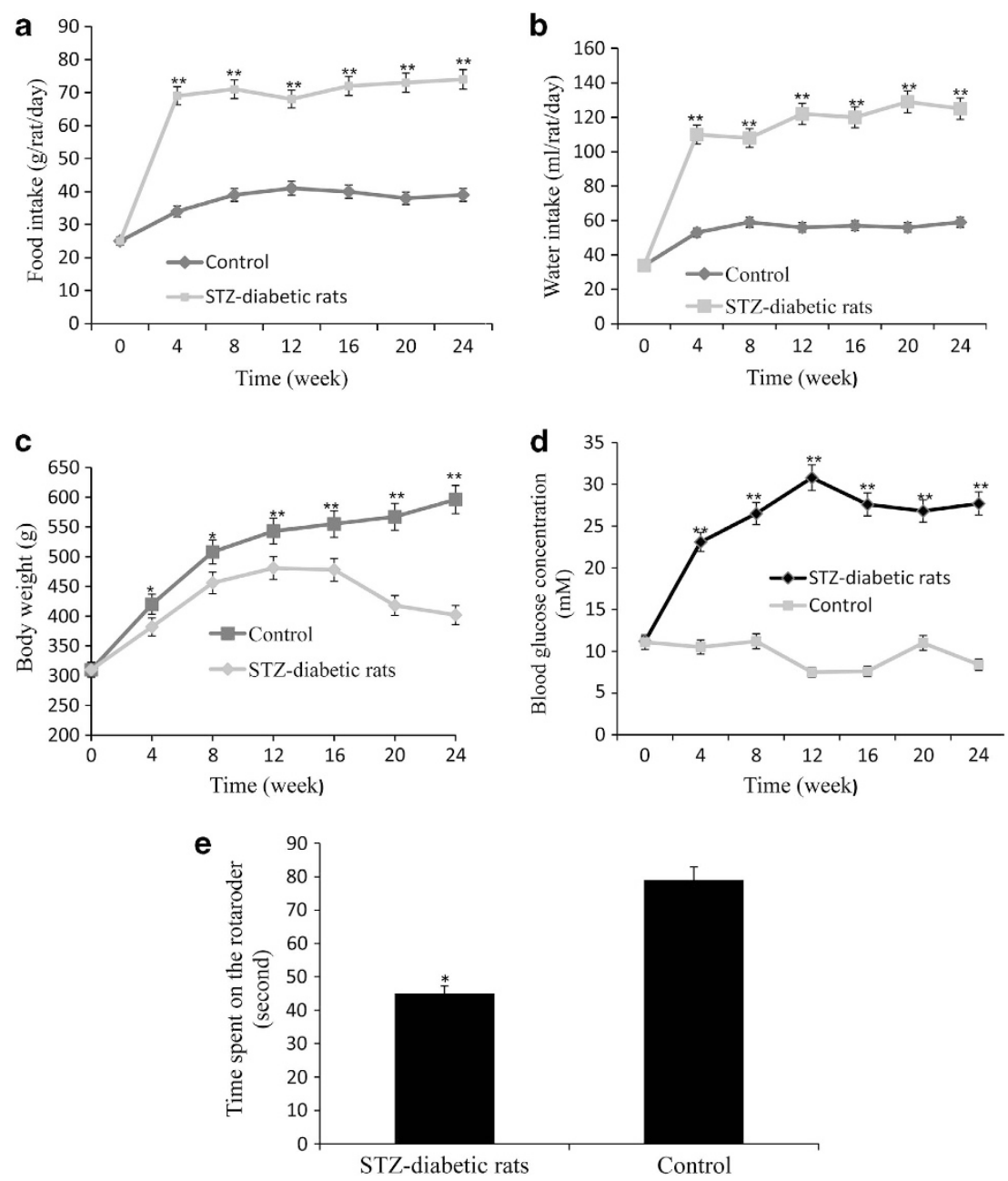

Figure 1 The intake of food and water, body weights, blood glucose, and motor coordination function in STZ-diabetic rats. (a) The food intake and (b) water intake of the STZ-diabetic rats ( $n=40 /$ group) compared with control rats $(n=25 /$ group). (c) The body weights and (d) blood glucose concentrations of the STZ-diabetic rats compared with control rats maintained for 24 weeks of observation (one-way ANOVA, ${ }^{*} P<0.05,{ }^{\star *} P<0.01$, significantly different from control rats). Error bars indicate mean \pm S.D. from three independent experiments. (e) Behavioral analysis of 24-week STZ-diabetic rats ( $n=9 /$ group) and control rats ( $n=8 /$ group) in rotarod test. STZ-diabetic and control rats were tested for three consecutive trials on the rotarod. Results were expressed as the time spent (in seconds) on the rotarod (one-way ANOVA, ${ }^{*} P<0.05$, significantly different from control rats). Data represent mean \pm S.D. from 3 to 4 independent experiments

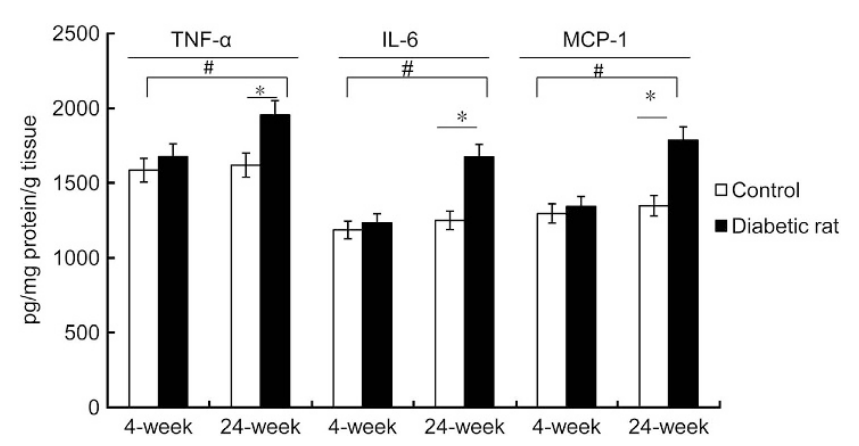

Figure 2 The TNF- $\alpha$, IL-6, and MCP-1 concentrations in the cerebella of STZdiabetic rats. The concentration of tumor necrosis factor (TNF)- $\alpha$, interleukin (IL)- 6 , and MCP-1 in STZ-diabetic rats at 4 and 24 weeks compared with controls $\left({ }^{\#} P<0.05,24\right.$-week diabetic rats versus 4-week rats; ${ }^{*} P<0.05$, 24-week diabetic versus 24-week control rats). Error bars indicate mean \pm S.D. from three independent experiments. Two-way ANOVA followed by the Newman-Keuls post hoc testing for pair-wise comparison was used for analysis of diabetic and control rats at two time points (4 and 24 weeks) containing LC3II-stained dots, and were devoid of any structures resembling autophagosomes particularly in axonal swellings and cell bodies (Figure $3 b$ ). The impaired autophagic activity was further confirmed by fluorescent intensity of p62 punctuate in Purkinje cells by immunoflurosence staining, with Calbindin acting as a Purkinje cell marker (Figure 3c). The quantification of the accumulation of p62 protein level aggregation in the deep cerebellar nuclei (DCN) area increased by $56.5 \%$ in the cerebella of STZ-diabetic rats ( $n=6$ /group) in contrast to that in controls ( $n=7 /$ group) $\left({ }^{\star \star} P<0.01\right.$; Figure $3 d$ ). Thus, the results suggested that dystrophic swellings of axon terminals in Purkinje cells were no longer able to generate an autophagic response in 24-week STZ-diabetic rats.

Loss of Purkinje cells in DCN of 24-week STZ-diabetic rats. To investigate the consequence of impaired autophagic activity on Purkinje cells in 24-week STZ-diabetic rats, midsagittal cerebella were stained with hematoxylin and eosin. 

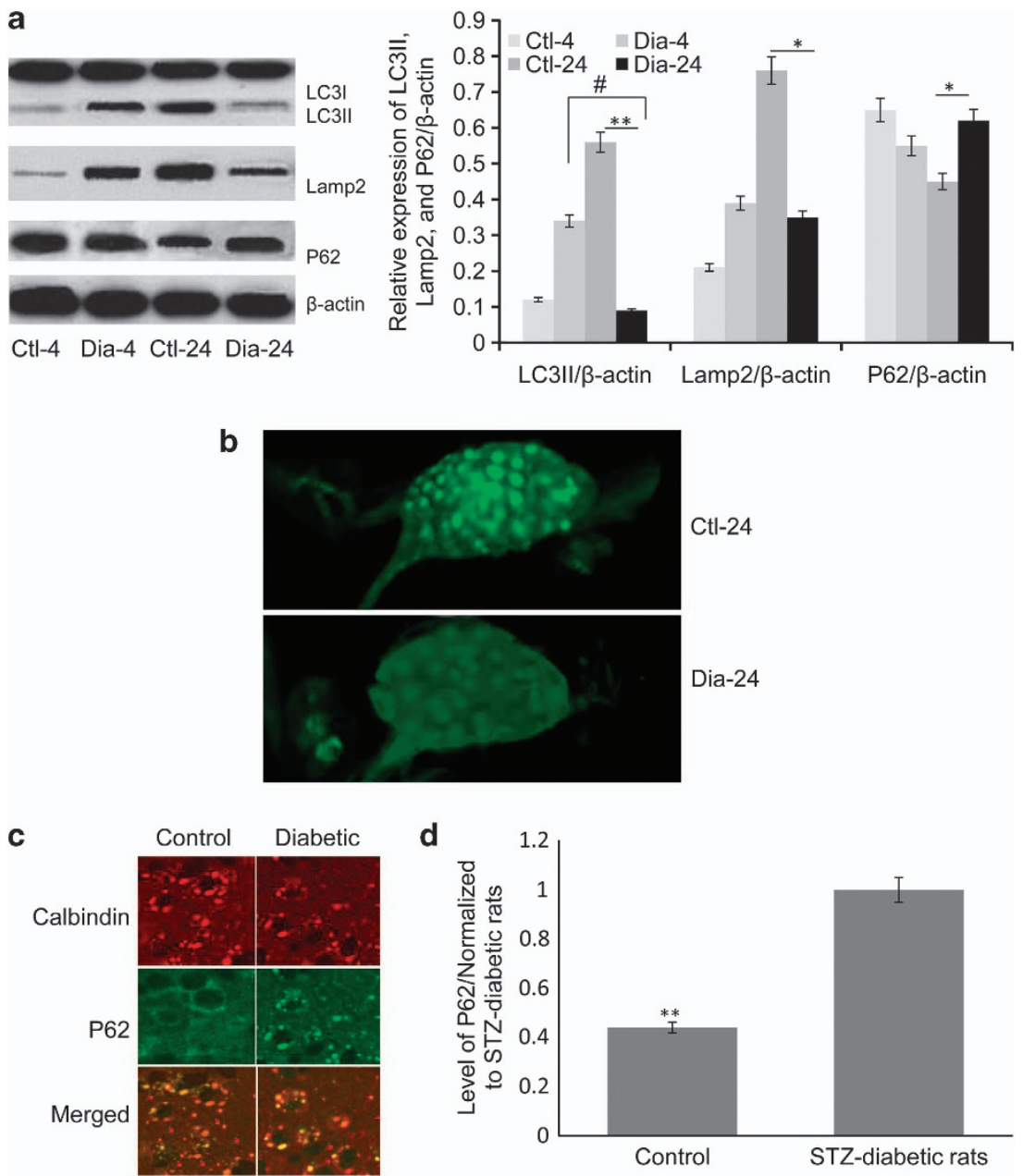

Figure 3 Autophagic activity protein expression, anti-LC3-labeled autophagosomes, and p62 accumulation in Purkinje cells of 24-week STZ-diabetic rats. (a) Autophagyassociated proteins LC3I, LC3II, Lamp2, and p62 levels were analyzed by immunoblotting at 4 and 24 weeks of STZ-diabetic rats compared with controls (Ctl-4, 4-week control rats; Ctl-24, 24-week control rats; Dia-4, 4-week diabetic rats, Dia-24, 24-week diabetic rats). The relative expression of LC3II, Lamp2, and p62/ $\beta$-actin is shown in the right panel $\left({ }^{\#} P<0.05\right.$, 24-week diabetic rats versus 4-week diabetic rats; ${ }^{*} P<0.05,{ }^{*} P<0.01$, 24-week diabetic versus 24 -week control rats). Mean \pm S.D. from three independent experiments. Two-way ANOVA followed by the Newman-Keuls post hoc testing for pair-wise comparison was used for analysis of diabetic and control rats at two time points (4 and 24 weeks). (b) Representative confocal images showed the redistribution of anti-LC3 green foci in the DCN of control ( $n=7 /$ group) (up) and 24-week STZ-diabetic rats ( $n=6 /$ group) (down). (c) Levels of p62 were exhibited in dystrophic swellings of Purkinje cells in 24-week STZ-diabetic rats. Anti-p62 immunofluorescent staining (in green) showed p62 in Purkinje cell axonal dystrophic swellings (Calbindin labeling in red). (d) Quantified levels of p62. The levels of p62 were normalized against values from STZ-diabetic rats (one-way ANOVA, ${ }^{* *} P<0.01$, 24-week diabetic versus 24-week control rats). Mean \pm S.D. from three independent experiments

Histological analyses indicated that STZ-diabetic rats had a severe defect in cerebellar Purkinje cells, and STZ-diabetic cerebellum degenerated in contrast to growth in the WT cerebellum during the 24-week period (Figure 4a). Next, we examined the Purkinje cells' morphology in STZ-diabetic rats by immunostaining of cerebellum with anti-NeuN (red staining for non-Purkinje cells) overlapping with confocal images (Figures $4 \mathrm{~b}-\mathrm{d}$ ), Figures $4 \mathrm{~h}-\mathrm{j}$, from control rats acting as a control. No staining above background was observed in Purkinje cells in both diabetic and control rats. The Purkinje cells were brightly stained by immunostaining of cerebellum with anti-Calbindin overlapping with confocal images (Figures $4 \mathrm{e}-\mathrm{g}$, Figures $4 \mathrm{k}-\mathrm{m}$ were from control rats). In 24-week diabetic rats, a striking degeneration of Purkinje cells labeled with Calbindin (red) (Figure 4f) compared with control (Figure 4I), was characterized by the presence of a noticeably decreased number of Purkinje cells in the DCN (Figure $4 \mathrm{~g}$ ) in comparison with control (Figure $4 \mathrm{~m}$ ). Purkinje cells were not recognized by anti-NeuN staining in STZ-diabetic (Figure 4c) or control rats (Figure 4i). The loss of Purkinje cells in STZ-diabetic rats was further confirmed by counting the number of Purkinje cells with Calbindin/Alexafluor488 (green) staining. The number of Purkinje cells in STZ-diabetic rats was markedly decreased in 24-week STZ-diabetic rats ( $n=6 /$ group) compared with normal rats ( $n=8 /$ group) (Figure $4 n$ ). The quantification of the loss of Purkinje cell number was reduced by $34.1 \%$ in the DCN area of STZ-diabetic rats cerebella compared with controls ( ${ }^{* *} P<0.01$; Figure 40 ), further supporting the onset of Purkinje cell degeneration in 24-week STZ-diabetic rats. Together, the existence of cell-autonomous degeneration and loss of Purkinje cells suggested the distinctive progression of neuropathy in 24-week diabetic rats. 
a

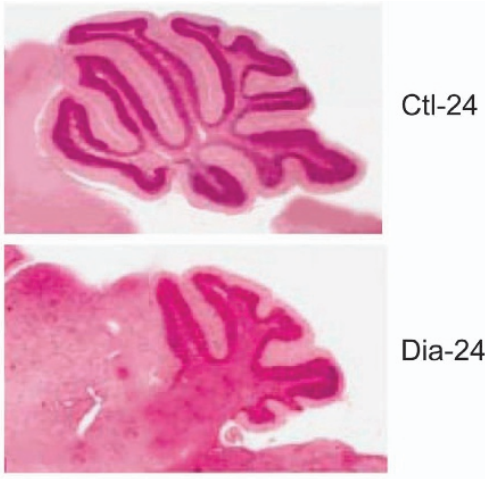

confocal images

anti-NeuN

overlapping
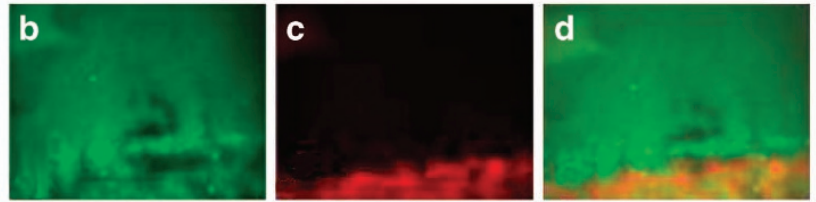

Diabetic

confocal images

anti-calbindin

overlapping
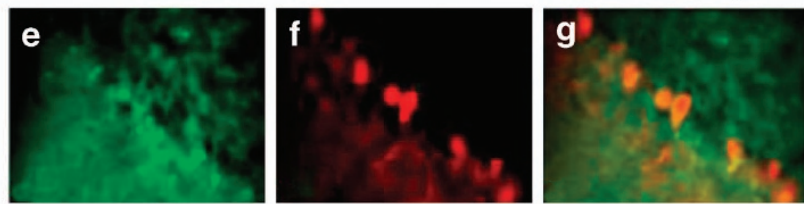

Diabetic

confocal images

anti-NeuN

overlapping
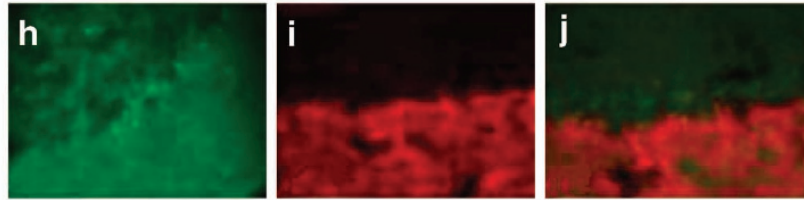

Control

contocal ımages

anti-calbindin
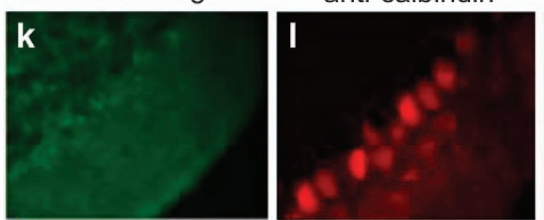

overlapping

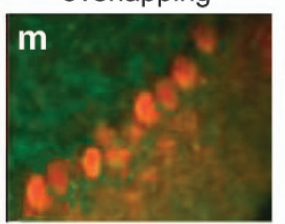

Control
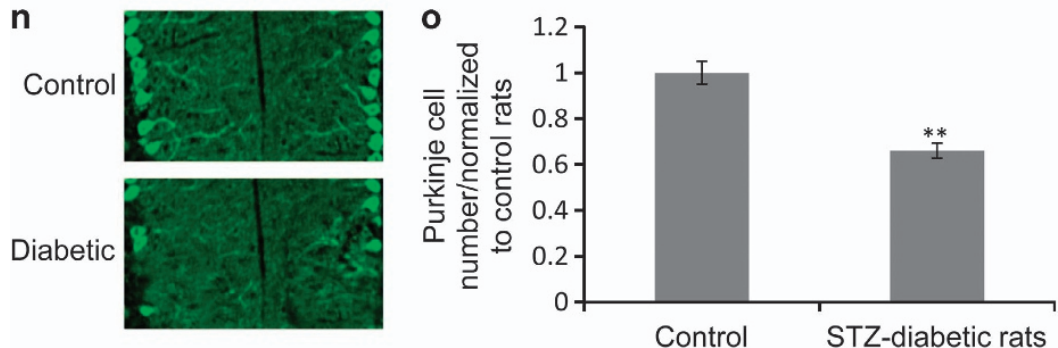

Figure 4 The degeneration and loss of Purkinje cells in 24-week STZ-diabetic rats. (a) Hematoxylin and eosin-stained midsagittal cerebella. (b and e) Representative confocal images showed the redistribution of green foci in the Purkije cells of STZ-diabetic rats ( $n=6 /$ group). (c) Immunofluorescent staining with monoclonal anti-NeuN antibody in STZ-diabetic rats. (f) Immunofluorescent staining with monoclonal anti-Calbindin antibody in STZ-diabetic rats. (d) Overlapping of green fluorescence with red immunofluorescent staining of monoclonal anti-NeuN in STZ-diabetic rats. (g) Overlapping of green fluorescence with red immunofluorescent staining of anti-Calbindin in STZ-diabetic rats. ( $\mathbf{h}$ and $\mathbf{k}$ ) Representative confocal images showed the redistribution of green foci in the Purkinje cells of control rats. (i) Immunofluorescent staining with monoclonal anti-NeuN antibody in control rats. (I) Immunofluorescent staining of monoclonal anti-Calbindin antibody in control rats. (j) Overlapping of green fluorescence with red immunofluorescent staining of monoclonal anti-NeuN in control rats. $(\mathbf{m})$ Overlapping of green fluorescence with red immunofluorescent staining of anti-Calbindin in control rats. (n) Representive presence of Purkinje cell loss in the DCN in two groups by Calbindin/Alexafluor488 (green). (o) Quantification of the images of panel (n). Bar, $20 \mu \mathrm{m}$. The Purkinje cell number was normalized against values from control rats. Differences between means were analyzed by one-way ANOVA. ${ }^{\star \star} P<0.01$, significantly different from control rats. Data represent mean \pm S.D. from 3 to 4 independent experiments 
Induction of higher expression LRRK2 in Purkinje neurons in 24-week STZ-diabetic rats. Given the fact that mutant LRRK2 with toxic kinase led to neuronal death and its functions were largely to stabilize microtubules at axon terminals, it was conceivable that LRRK2 may have a role in diabetic neuropathy. ${ }^{36}$ To investigate whether LRRK2 expression level changed in dystrophic and degenerative axonal swellings of Purkinje cells in STZ-diabetic rats, we performed in situ hybridization with cerebellar slices as shown in Materials and Methods. Although cerebellar cortex in controls showed normal staining of LRRK2, the cerebellar cortex of STZ-diabetic rats showed Purkinje cells that were much detectably stained (Figure 5a). The expression level of LRRK2 was further studied using the brain extracts of STZ-diabetic rats via western blotting analysis (Figure $5 b$ ). The result showed that 24-week STZ-diabetic rats ( $n=6$ /group) produced large amounts of LRRK2 compared with 24-week control rats ( $n=6 /$ group) (Figure $5 c$ ). The quantification of relative LRRK2 expression level was significantly increased in 24-week diabetic rats compared with 24-week control rats $\left({ }^{\star} P<0.05\right)$ and was markedly higher than that in 4-week rats $(\# P<0.05)$. The results suggested a higher expression of LRRK2 in dystrophic axon terminals of Purkinje cells in diabetic rats.

\section{Purkinje cell loss driven by LRRK2-mediated hyper-} phosphorylation of tau along with increased mito-DLP1 in 24-week diabetic rats that caused mitochondrial dysfunction in vitro. It was reported that LRRK2 interacted with tau in a tubulin-dependent manner, and LRRK2mediated phosphorylation of tau reduced its tubulin-binding ability. ${ }^{37}$ To investigate whether excessive LRRK2 expression phosphorylated tubulin-associated tau in 24-week STZ-diabetic rats, we performed immunoblotting to measure the LRRK2, total tau, and phosphorylated tau as shown in Materials and Methods to test whether LRRK2-mediated phosphorylation of tau correlated with LRRK2 levels in these 24-week STZ-diabetic rats. ${ }^{6}$ Western blotting analysis with an antibody revealed that LRRK2 protein expression in 24-week STZ-diabetic rats was much higher than that in controls (Figure 6a). Along with this, 24-week STZ-diabetic rats exhibited a 1.7-fold higher tau phosphorylation compared with controls. Thus, the phosphorylated tau level was significantly increased in 24-week STZ-diabetic rats associated with the elevation level of LRRK2 protein ( ${ }^{\star} P<0.05$; Figure 6b), suggesting LRRK2-mediated hyperphosphorylation of tau in 24-week STZ-diabetic rats. Next, to investigate whether hyperphosphorylation of tau along with increased DLP1 led to mitochondrial fragmentation in Purkinje cells, which had been associated with cell apoptosis in vitro, we performed immunoblot and quantitative analysis of mito-DLP1 expression. The data revealed that mitochondrial DLP1 was increased significantly in 24-week STZdiabetic rats compared with controls $\left({ }^{\star} P<0.05\right.$; Figures $6 \mathrm{c}$ and d). Next, we investigated the effects of LRRK2 on the mitochondrial morphology. Representative electron microscopy showed fragmented mitochondria in LRRK2overexpressing cells compared with predominant tubular mitochondrial morphology in control (Figure 6e), suggesting that LRRK2-mediated rise of mitochondrial DLP1/P-tau induced more mitochondrial fragmentation. To investigate whether the mitochondrial dysfunction in vitro was driven by LRRK2 overexpression, we measured the mitochondrial protein degradation in cultured cerebellar Purkinje neurons after LRRK2 transfection. We examined the rate of mitochondrial protein of SDHA subunit of complex II (CII), ATP synthase (CV), barrel protein (porin), and integral membrane protein (Tom20) degradation by transfecting cells

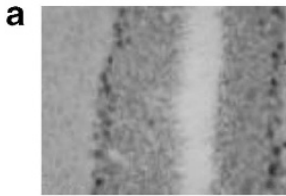

Ctl-24

C

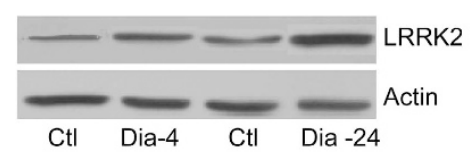

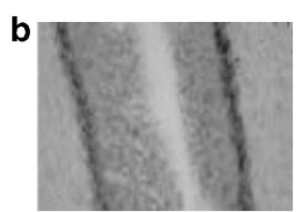

Dia-24

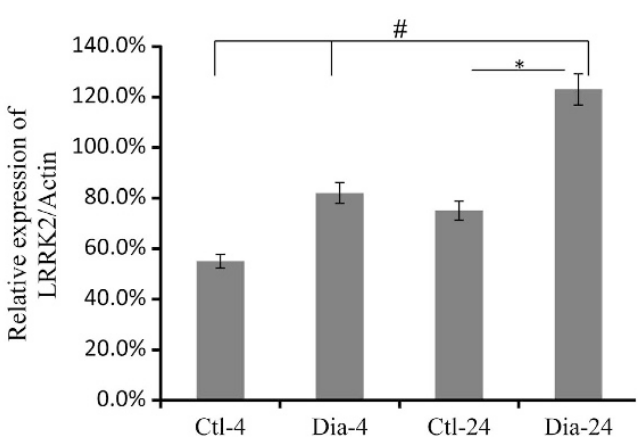

Figure 5 The distribution and expression of LRRK2 in the brain of STZ-diabetic rats. (a and $\mathbf{b}$ ) Neuroanatomical distribution of LRRK2 mRNA was detected in Purkinje cells of 24-week STZ-diabetic and control rats, respectively. Cerebellar cortex rat sections were hybridized with a digoxigenin-labeled riboprobe complementary to rat LRRK2 and further processed as described in the Materials and Methods section. (c) LRRK2 expression of Purkinje cells from 4-week STZ-diabetic ( $n=6 /$ group), 24-week STZ-diabetic ( $n=6 /$ group), and control rats were detected by western blotting. Densitometry values represented the ratio of LRRK2/Actin was showed in the right panel $\left({ }^{\prime} P<0.05,24\right.$-week diabetic rats versus 4-week control rats; ${ }^{*} P<0.05$, 24-week diabetic versus 24 -week control rats). Error bars indicate mean \pm S.D. from three independent experiments. Two-way ANOVA followed by the Newman-Keuls post hoc testing for pair-wise comparison was used for analysis of diabetic and control rats at two time points (4 and 24 weeks) 
with the LRRK2-inserted vector. As a result, cells with LRRK2 demonstrated a lower rate of mitochondrial protein degradation compared with controls. Effects of LRRK2 transfection on protein levels of other cellular compartments such as Golgi (Golgi-58), lysosome (Lamp2), endoplasmic reticulum (calregulin), or cytosol (Gapdh, Actin) were independent of LRRK2 effects (Figure 6f), suggesting that only mitochondrial protein degradation rate was impacted by LRRK2 overexpression.

The rise of LRRK2 expression impaired mitochondrial function in 24-week STZ-diabetic rats. To examine a

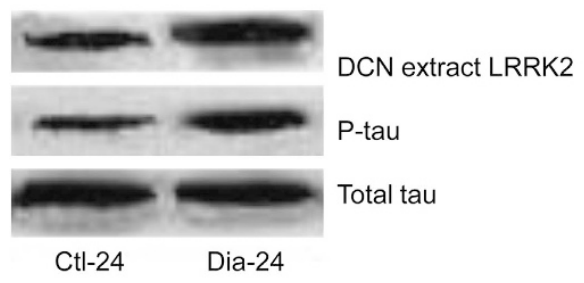

C

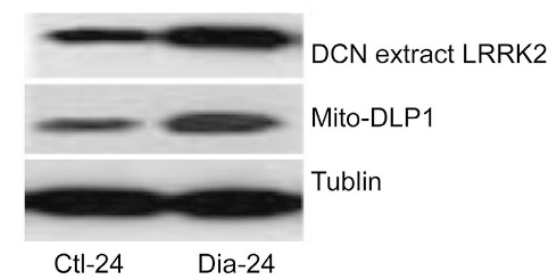

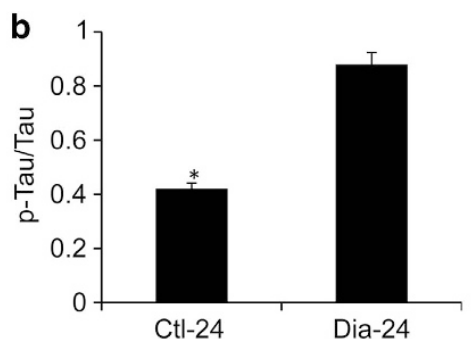

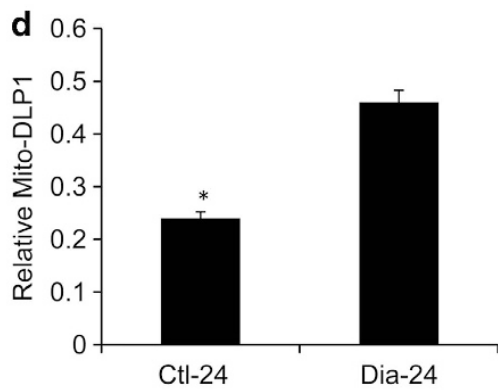

e

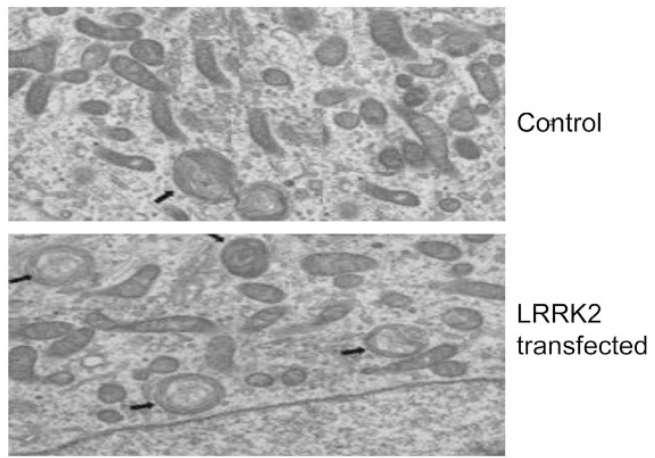

f

Ctl, LRRK2 transfected
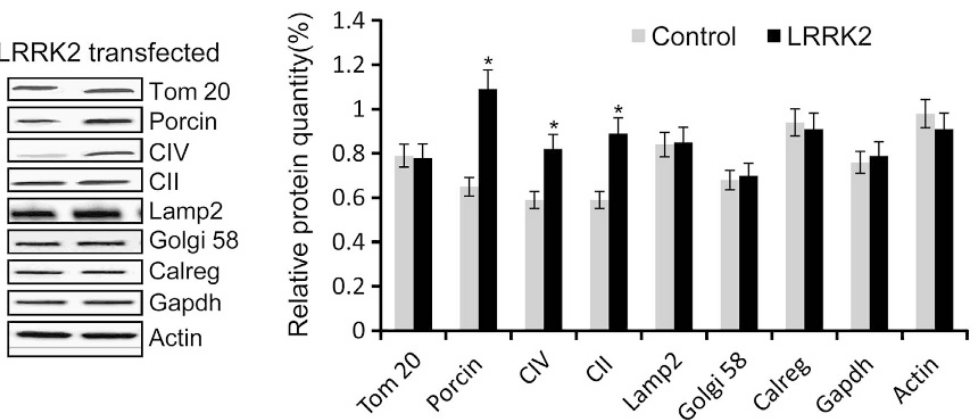

Figure 6 The effect of LRRK2 overexpression on phosphorylation of tau and mito-DLP1 levels in vivo, mitochondrial morphology, and protein degradation in vitro. (a) Proteins were analyzed using antibodies against LRRK2, phospho-tau, and non-phosphorylated tau. (b) The ratio of P-tau/tau is shown in the right panel (one-way ANOVA, $\left.{ }^{*} P<0.05\right)$. (c) Representative immunoblot and (d) quantification analysis levels of mito-DLP1 in diabetic rats compared with controls (one-way ANOVA, $\left.{ }^{*} P<0.05\right)$. (e) Representative electron microscopy analysis of multilamellar onion-like structures in cultured cerebellar Purkinje neurons transfected by LRRK2 compared with controls, arrows marked the mitophagy inside cells. At least 30 mitochondria were analyzed. (f) Mitochondrial proteins were measured in cultured cerebellar Purkinje neurons transfected by LRRK2-inserted PCMV-AC-GFP vectors. Level of the mitochondrial proteins was analyzed using antibodies against complex II (CII), ATP synthase (CV), porin, and Tom20. Golgi apparatus, cytosol, lysosomes, and endoplasmic reticulum were analyzed using Golgi58, Gapdh, Lamp2, and calregulin (calreg) antibodies. Quantification of protein level is shown in the right panel. Black bars are LRRK2, and gray bars are controls (one-way ANOVA, ${ }^{*} P<0.05$, significantly different from control cells). Data are presented as mean \pm S.D. from 3 to 4 independent experiments 
whether mitochondrial dysfunction occurred in Purkinje neurons from diabetic rats, organotypic cerebellar slice cultures from diabetic and control rats were labeled with 5,5',6,6'-tetrachloro-1,1',3,3'-tetraethylbenzimidazolylcarbocyanine iodide (JC-1), a membrane permeable dye that emits in the green spectra in mitochondria with a low $\Delta \psi \mathrm{m}$ but fluoresces red in the presence of a large $\Delta \psi \mathrm{m}$. Cultures from control rats had a higher red: green ratio than diabetic rats (Figure 7a). Because LRRK2 has an important role in mitochondrial homeostasis, ${ }^{38}$ it is conceivable that the mitochondria will be functionally impaired under LRRK2 overexpression in cerebellar Purkinje neurons. To investigate whether LRRK2 overexpression induced damaged mitochondrion-producing ROS, we measured mtROS production by three types of mitochondria-specific labels that distinguish respiring (Mitotracker deep red), total (Mitotracker green), and ROS-generating mitochondria (MitoSOX) in these diabetic and control rats. We found that LRRK2 overexpression resulted in loss of mitochondrial membrane potential and robust ROS production in diabetic rats (Figure $7 \mathrm{~b}$ ). Taken together, our data indicated that LRRK2 overexpression had a deleterious role in mitochondrial morphology and function in 24-week STZ-diabetic rats.

\section{Discussion}

Diabetic neuropathy is the most common complication and the greatest source of morbidity and mortality in diabetes patients. There is now increasing evidence of CNS involvement in diabetic neuropathy. ${ }^{14}$ Several findings emerge from our study and are as follows: (1) 24-week STZ-diabetic rats showed motor coordination dysfunction; (2) elevation level of proinflammatory cytokines and chemokines in 24-week STZ-diabetic rats indicated that inflammation activation was implicated in the progression of diabetic neuropathy; (3) impaired autophagic activity, cell-autonomous dystrophy, and degeneration in the Purkinje cells of 24-week STZ-diabetic rats implied a neuroprotective role of autophagy in the progression of diabetic neuropathy; ${ }^{27,28}$ (4) no autophagosome formation and aggregation of p62 reflected failure of autophagy function changes of Purkinje cells in 24-week STZ-diabetic rats; (5) degeneration and loss of Purkinje cells of the 24-week STZ-diabetic rats were driven by an increased LRRK2-mediated hyperphosphorylation of tau along with elevation of mito-DLP1; (6) LRRK2 overexpression induced mitochondrial fragmentation and blocked mitochondrial protein degradation in vitro; and (7) higher expression of LRRK2 in cerebellar neurons impaired mitochondrial function in 24-week STZ-diabetic rats. The evidence suggests that LRRK2-mediated mitochondrial dysfunction and loss of cerebellar Purkinje neurons is a novel mechanism underlying the pathogenesis of diabetic neuropathy. ${ }^{39}$

LRRK2 gene is active in the brain and other tissues throughout the body. In this study, degeneration and loss of Purkinje cells in 24-week STZ-diabetic rats were driven by an increased LRRK2-mediated hyperphosphorylation of tau a
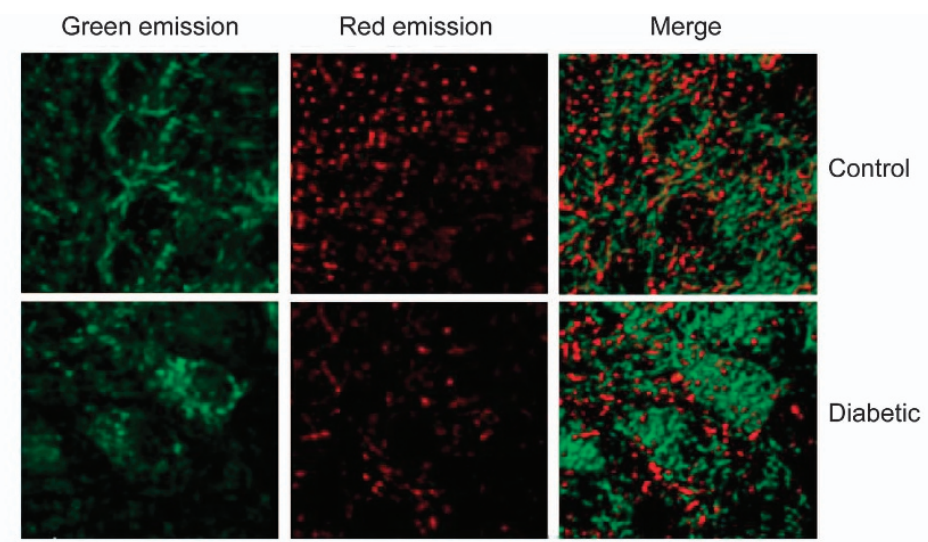

b

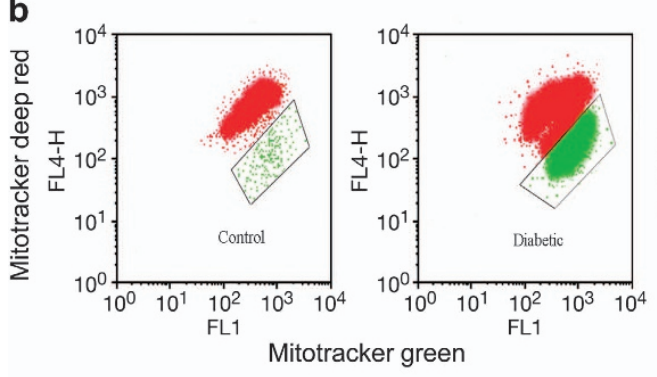

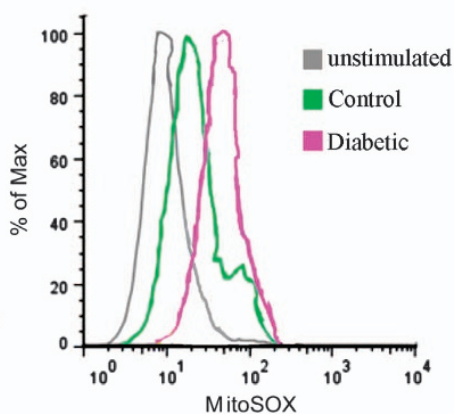

Figure 7 The mitochondrial function in cerebellar Purkinje neurons of 24-week STZ-diabetic rats. (a) Immunofluorescence panels of cerebellar slice cultures from 24-week STZ-diabetic and control rats. Cultures were treated with JC-1, a cationic dye that exhibits green emission in weakly polarized mitochondria that shifts to red in more strongly depolarized mitochondria. Optical sections of 300-400 nm were taken, line-averaged twice to improve the signal-to-noise ratio, and image stacks were deconvolved by using the Huygens Essential software (scale bar: $10 \mu \mathrm{m}$ ). (b) Cerebellar Purkinje neurons from 24-week STZ-diabetic and control rats were stained with Mitotracker green and Mitotracker deep red or MitoSOX for 30 min and analyzed by flow cytometry 
along with elevation of mito-DLP1, and LRRK2 overexpression-impaired mitochondrial function both in vitro and in vivo. These results were congruent with a previous report that LRRK2 expression level was relevant to the autophagic activity and counterbalanced the effect on autophagy-regulated cell death under the stressful condition of starvation. ${ }^{40-}$ Tau is a microtubule-associated protein and a physiological substrate for LRRK2 found predominantly in the CNS and expressed mainly in neuronal axons. Tau drives neurite outgrowth by promoting the assembly of microtubules, which is critical for the establishment of neuronal cell polarity. ${ }^{41}$ LRRK2 overexpression-mediated phosphorylated tau forming a paired helical filament could lead to tau-based neurofibrillary lesions, the predominant brain pathology previously referred to as 'tauopathy'. ${ }^{42}$ In agreement with a previous study, our results demonstrated that higher expression LRRK2 in 24-week STZ-diabetic rats led to a corresponding elevation of phosphorylation tau change involved in Purkinje cell loss. Why does higher expression of the LRRK2-mediated pathway result in mitochondrial dysfunction and cell loss in cerebellar Purkinje neurons of STZ-diabetic rats? Evidence has been accumulating that the deleterious effect of hyperglycemia can be detrimental to cells in the cerebellum. Purkinje cells have recently been shown to be highly susceptible to autophagy induction under various stress conditions. ${ }^{27,28}$ Harmful imbalances in glucose tolerance in STZ-diabetic rats are conceptualized as a state of oxidative stress. Neurons may be particularly vulnerable to developing oxidative stress, in part, due to the need for transport along neurites for long distances. ${ }^{27}$ The discovery of the potential role of mitochondria in diabetic neuropathy in our study has been suggested to be another reason. Our findings showed that higher LRRK2 expression in Purkinje cells damaged mitochondrial function in STZ-diabetic rats and was further confirmed in cultured cerebellar Purkinje neurons transfected by LRRK2 overexpression. The mitochondria in these diabetic rats are especially vulnerable, because hyperglycemic neurons are the origin of production of ROS, which can damage their DNA and membranes. Deregulation of fission and fusion proteins that control mitochondrial shape and number can impair cell functions and might lead to cell degeneration. ${ }^{43}$ Third, LRRK2 is specifically found to be involved in the regulation of DLP1-dependent mitochondrial fission by increasing mitochondrial DLP1 recruitment through increased interaction with DLP1 in vitro. Given that LRRK2 was highly expressed in these diabetic rats, it was not surprising to find that, in our study, LRRK2 elevation enhanced mitochondrial fragmentation. Finally, as mentioned above, LRRK2 has an important role as a physiological regulator for phosphorylation-mediated dissociation of tau from microtubules, which is an integral aspect of microtubule dynamics that are essential for mitophagy. ${ }^{37}$ Abnormal hyperphosphorylation of microtubule-associated protein tau by higher LRRK2 expression could lead to mitochondrial motility arrest, essential for mitophagy activation and following cell apoptosis. Combined with previous reports, our data demonstrated a novel cellular mechanism of LRRK2-mediated mitochondrial dysfunction and loss of cerebellar Purkinje neurons in diabetic neuropathy. It would be interesting to investigate how mitophagy regulated by
LRRK2 as its particular localization to outer membrane of mitochondria in future.

Patients with diabetes present with proximal neuropathy of the lower limbs, characterized by a variable degree of pain and sensory loss, and experience difficulty in walking and climbing stairs. ${ }^{44}$ Our data showed alteration of motor function in 24-week STZ-diabetic rats by the rotarod test. Whether higher expression of LRRK2-mediated mitochondrial dysfunction and cell loss in cerebellar Purkinje neurons causes motor coordination deficits remains to be established in future. Thus, the emerging evidence, including the present findings, suggests that modulating LRRK2 function could help restore the altered mitochondria homeostasis balance and lead to new treatments and neuroprotection strategies. It would be important either to manipulate LRRK2 activity genetically or to pharmacologically establish cause and effect in a diabetic rat model for future research. Significant progress in this field will provide valuable knowledge to the design of drugs targeted at LRRK2 in the treatment of diabetic neuropathy diseases.

\section{Materials and Methods}

Antibodies. Antibodies used as secondary antibodies were anti-Calbindin (1:250; Sigma, St. Louis, MO, USA; Cat. No. C2724), anti-LC3B (1:500; Sigma, L7543), anti-LAMP2 (1:300; Abcam, Cambridge, MA, USA; Cat. No. ab13524), anti-p62 (1:200; American Research Products, Waltham, MA, USA; Cat. No. 03-GP62-C), Cy3-conjugated anti-rabbit IgG (1:2000; Upstate Biotechnology, New York, NY, USA; Cat. No. 715-095-150), anti-NeuN (1:100; Millipore, Billerica, MA, USA; Cat. No. ABN90), anti-tau (1:1000; Abcam, Cat. No. ab64193), anti-phosphorylated tau (1:200; Thermo Fisher Scientific, Lenexa, KS, USA; Cat. No. AT270), anti-DLP1 (1: 1000; Abcam, Cat. No.ab156951), anti-actin (1:2000; Abcam, Cat. No. AB8277), anti-LRRK2 MJFF4 (1:2000; Epitomics, Burlingame, CA, USA; Cat.No. 3516), anti-Tom20 (1:250; SantaCruz, Dallas, TX, USA; Cat. No. sc-17764), anti-calregulin (1:250; SantaCruz, Cat. No. sc-6468-R), anti-Gapdh (1:2500; Abcam, Cat. No. ab9483), anti-Golgi 58 (1:5000; Thermo Fisher Scientific, Cat. No. MA1-22144), and Alexa Fluor-conjugated antibodies (1: 2000; Molecular Probes, Eugene, OR, USA; Cat. No. A21057, A21076, A21096).

Cell culture systems. Twenty-four-week diabetic and control rats' cerebellar Purkinje neurons were prepared as described and maintained in neurobasal medium supplemented with B-27, N-2, L-glutamine $(500 \mu \mathrm{m})$, and penicillin/ streptomycin (100 units/ml) (Invitrogen, Carlsbad, CA, USA).

Cell transfection. Cultured cerebellar Purkinje neurons were transfected as described in the text with Lipofectamine 2000 reagent (Invitrogen) according to the manufacturer's suggested protocol. In the experiment to study LRRK2 overexpression-induced mitochondrial dysfunction, cells were transfected with LRRK2-inserted pCMV6-AC-GFP vector (OriGene, Rockville, MD, USA).

Rat. Wistar rats were fed ad libitum with commercial pelleted chow (UAR, Villemoisson-sur-Orge, France; carbohydrate $47 \%$, protein $20 \%$, fat $8 \%$ ). Insulin deficiency was induced by an i.p. injection of $80 \mathrm{mg} / \mathrm{kg}$ of STZ diluted in $30 \mu \mathrm{l}$ of citrate buffer $(0.05 \mathrm{M}, \mathrm{pH} 4.5)$ and administered on the fifth day after birth to develop T2D as reported previously. ${ }^{45,46}$ All the rats that received only $30 \mu \mathrm{l}$ of citrate buffer on the fifth day after birth were used as controls. Diabetic rats were given $1 \mathrm{U}$ of long-acting insulin (Ultralente, Novo Nordisk, Bagsvaerd, Denmark) daily to maintain body weight and to prevent ketoacidosis without normalizing hyperglycemia. The success rate of inducing diabetes (defined as a blood glucose concentration of $15 \mathrm{mM}$ and a water intake of $100 \mathrm{ml} /$ rat/day) was approximately $90 \%$. All control age-matched rats $(n=25)$ survived the 24-week period. Sixty pecent of STZ-diabetic rats $(n=24)$ survived for 24 weeks. Brain inflammatory cytokines were detected at the 4-week and 24-week time point. The intake of food and water and body weight were measured, and blood glucose concentrations were monitored every 4 weeks. Both the diabetic and control rats were euthanized after 24 weeks. The brains were excised, washed in saline, and stored at $-80^{\circ} \mathrm{C}$ until analysis. All experimental procedures were approved by the Nanjing Normal University Animal Care and Use Committee and 
were conducted in full accordance with the Guide for the Care and Use of Laboratory Animals.

Analysis of proinflammatory biomarkers. The cerebellum dissected from rat brain was described previously. ${ }^{47}$ The cerebella(g tissue) were homogenized in a lysis buffer that contained $50 \mathrm{mM}$ tris (hydroxymethyl) aminomethane ( $\mathrm{pH} 8.0), 150 \mathrm{mM}$ sodium chloride, and 1\% 4-nonylphenylpolyethylene glycol (Nonidet P-40, Sigma-Aldrich, St. Louis, MO, USA). The homogenate was centrifuged at $5000 \times g$ for $15 \mathrm{~min}$ at $4{ }^{\circ} \mathrm{C}$ for isolation of total supernatant protein. Protein concentration ( $\mathrm{mg}$ protein) was determined with Pierce BCA Protein Assay Kit (Thermo Scientific Pierce Protein Biology Products, Rockford, IL, USA) according to the instructions of the manufacturer. The levels of TNF- $\alpha$, IL-6, and MCP-1 (pg cytokines) in the cerebellum were quantified using solid-phase sandwich ELISA (TNF- $\alpha$, IL-6, and MCP-1) kits (BioSource International, Inc., Camarillo, CA, USA). The concentrations were expressed as $\mathrm{pg}$ cytokines/mg protein/g tissue.

In situ hybridization histochemistry. Antisense and sense digoxigeninlabeled riboprobes were produced using $\mathrm{T} 3$ and $\mathrm{T} 7$ transcription systems and $1 \mu \mathrm{g}$ of linearized plasmid as previously described. Riboprobes were recovered in distilled water and stored in aliquots at $-70^{\circ} \mathrm{C}$ until use. Sections to be hybridized were described previously. ${ }^{48}$

Fluorescence microscopy. Diabetic and control rats were anesthetized with $50 \mathrm{mg} / \mathrm{ml} \mathrm{Nembutal,} 0.1 \mathrm{cc}$ for adult. The whole-body perfusion was performed with $4 \%$ paraformaldehyde in PBS, pH 7.4, using a peristaltic pump (Rainin Instruments, Oakland, CA, USA; Cat. No. P757). The perfused brain tissues were postfixed again in $4 \%$ paraformaldehyde for $24 \mathrm{~h}$ at $4{ }^{\circ} \mathrm{C}$ and embedded in $5 \%$ low-melting agarose gel. Sagittal brain slices of $60 \mu \mathrm{m}$ thickness were prepared using Tissue Sectioning and Bath Refrigeration Systems (Vibratome, St. Louis, MO, USA; Cat. No. A11073) and were stored in PBS at $4{ }^{\circ} \mathrm{C}$. Brain slices were blocked with PBS containing $0.05 \%$ Triton X-100 and $10 \%$ goat serum and then incubated with the primary antibody of interest (anti-p62, anti-NeuN, and anti-Calbindin as appropriate) at $4{ }^{\circ} \mathrm{C}$ overnight. The slices were washed and incubated with Cy3-conjugated anti-rabbit or anti-rabbit Alexa Fluor as appropriate for $45 \mathrm{~min}$ at room temperature, washed extensively, mounted with ProLong Gold antifade reagent (Carlsbad, CA, USA), and examined using a Zeiss (Göttingen, Germany, Cat. No. 71878) confocal microscope. For statistical analysis of the Purkinje cell loss in the DCN area, confocal images were acquired with a $\times 60$ water objective lens. The number of Purkinje cells was counted for every view field.

Western blotting analysis. STZ-diabetic and control rats' tissues or cell lysates were separated by electrophoresis before transfer to PVDF membranes. Total protein extracts were obtained by homogenization in a TES buffer supplemented with a mixture of protease inhibitors (Complete; Roche Applied Science, Upper Bavaria, Germany; Cat. No.10324). Protein extracts (20 $\mu \mathrm{g}$ each) were fractionated on proper concentration of SDS-PAGE and blotted on a nitrocellulose membrane (Whatman, Maidstone, UK; Cat. No.34000). Immunoblots were blocked in $5 \%$ dry milk in TBST buffer $(10 \mathrm{mM}$ Tris, pH 7.5, $0.15 \mathrm{M} \mathrm{NaCl}$, $0.1 \%$ Tween 20) incubated with primary antibody of interest (anti-LC3B, antiLAMP2, anti-p62, anti-tau, anti-phosphorylated tau, anti-DLP1, anti-actin, antiLRRK2 MJFF4, anti-Tom20, anti-calregulin, anti-Gapdh, anti-Golgi 58 as appropriate). Anti-actin was used as an internal control. Bound antibodies were visualized with horseradish peroxidase-conjugated secondary antibodies and enhanced chemiluminescence (GE Healthcare, Buckinghamshire, UK; Cat. No. X1900) and exposure to hyperfilm (GE Healthcare, Cat. No. RPN1674K). ImageQuant software 5.0 (GE Healthcare, Cat. No. 27-0901-02) was used to determine the optical density of protein bands, and all data were normalized to the expression of $\beta$-actin.

Electron microscopy. Cerebellar Purkinje neurons transfected by LRRK2 compared with controls were cultured on the Aclar embedding film (2-mm thickness, Electron Microscopy Sciences, Hatfield, PA, USA), fixed in $2.5 \%$ glutaraldehyde and $4 \%$ sucrose in a $0.05 \mathrm{~mol} / /$ phosphate buffer, $\mathrm{pH} 7.4$, and examined with a JEOL 1200EX electron microscope (JEOL, Tokyo, Japan) as described previously. ${ }^{49}$

Mitochondrial isolation. Mitochondrial isolation was used to detect complex II (CII), ATP synthase (CV), porin, and Tom20 using mitochondrial isolation kit according to the manufacturer's instructions (Pierce, Rockford, IL, USA). Briefly, cells were homogenized in a dounce homogenizer and then centrifuged at $750 \times g$ for $10 \mathrm{~min}$ at $4^{\circ} \mathrm{C}$. The supernatant was further centrifuged at $12000 \times g$ for $15 \mathrm{~min}$ at $4{ }^{\circ} \mathrm{C}$. The pellet was then washed and kept as the mitochondrial fraction. The supernatant was further centrifuged at $100000 \times g$ for $1 \mathrm{~h}$ at $4{ }^{\circ} \mathrm{C}$ and designated as the cytosolic fraction.

Flow cytometry. MitoSOX (ROS-generating mitochondria), MitoTracker Green (total), Red (respiring), and DCFDA staining were performed according to the manufacturer's instructions (Invitrogen). Data were acquired with a FACSCalibur flow cytometer (BD Biosciences, Franklin Lakes, NJ, USA).

Rotarod test. Motor coordination and balance were evaluated by rotarod test. We performed the rotarod test using an accelerating rotarod by placing a rat on a rotating drum $(3 \mathrm{~cm}$ diameter) and measuring the time for which each animal was able to maintain its balance on the rod as latency time to fall (seconds). Speed of the rotarod was accelerated from 4 to 40 r.p.m. over a 5-min period. STZ-diabetic and control rats that fell were restarted for a total of three consecutive trials. The trials were used for statistical analysis.

Statistics. The data are presented as the mean \pm S.D. of three independent experiments unless otherwise noted. Differences between means were analyzed using either one-way or two-way ANOVA followed by the Newman-Keuls post hoc testing for pair-wise comparison using SPSS (Chicago, IL, USA). The null hypothesis was rejected when the $P$-value was $<0.05$.

\section{Conflict of Interest}

The authors declare no conflict of interest.

Acknowledgements. This study was supported by the 'National Natural Science Foundation of China (J1103507), Jiangsu NSF (BK2011784)' and 'The Priority Academic Program Development of Jiangsu Higher Education Institutions (PAPD)'.

\section{Author contributions}

SJ wrote the manuscript and researched data. $L L$ and $C X$ researched data. LZ contributed to discussion and reviewed/edited the manuscript. YH researched data and contributed to the discussion. SJ and LZ reviewed the manuscript.

1. Roman de Mettelinge T, Delbaere K, Calders P, Gysel T, Van Den Noortgate N, Cambier $D$. The impact of peripheral neuropathy and cognitive decrements on gait in older adults with type 2 diabetes mellitus. Arch Phys Med Rehabil 2013; 94: 1074-1079.

2. Di Domenico F, Sultana R, Ferree A, Smith K, Barone E, Perluigi M et al. Redox proteomics analyses of the influence of co-expression of wild-type or mutated LRRK2 and Tau on C. elegans protein expression and oxidative modification: relevance to Parkinson disease. Antioxid Redox Signal 2012; 17: 1490-1506.

3. Lin CH, Tsai PI, Wu RM, Chien CT. LRRK2 G2019S mutation induces dendrite degeneration through mislocalization and phosphorylation of tau by recruiting autoactivated GSK3ss. J Neurosci 2010; 30: 13138-13149.

4. Melrose HL, Dachsel JC, Behrouz B, Lincoln SJ, Yue M, Hinkle KM et al. Impaired dopaminergic neurotransmission and microtubule-associated protein tau alterations in human LRRK2 transgenic mice. Neurobiol Dis 2010; 40: 503-517.

5. Miklossy J, Qing H, Guo JP, Yu S, Wszolek ZK, Calne D et al. Lrrk2 and chronic inflammation are linked to pallido-ponto-nigral degeneration caused by the N279K tau mutation. Acta Neuropathol 2007; 114: 243-254.

6. Kawakami F, Yabata T, Ohta E, Maekawa T, Shimada N, Suzuki M et al. LRRK2 phosphorylates tubulin-associated tau but not the free molecule: LRRK2-mediated regulation of the tau-tubulin association and neurite outgrowth. PLoS One 2012; 7: e30834.

7. Braun AP. Convergent signaling events underlie dopamine-mediated inhibition of CaV3.2 T-type calcium channels. Channels 2009; 3: 381-382.

8. Sarantis K, Matsokis N, Angelatou F. Synergistic interactions of dopamine D1 and glutamate NMDA receptors in rat hippocampus and prefrontal cortex: involvement of ERK1/2 signaling. Neuroscience 2009; 163: 1135-1145.

9. Lebel M, Patenaude C, Allyson J, Massicotte G, Cyr M. Dopamine D1 receptor activation induces tau phosphorylation via cdk5 and GSK3 signaling pathways. Neuropharmacology 2009; 57: 392-402.

10. Kim YS, Shin JH, Hall FS, Linden DJ. Dopamine signaling is required for depolarizationinduced slow current in cerebellar Purkinje cells. J Neurosci 2009; 29: 8530-8538. 
11. Vincent AM, Callaghan BC, Smith AL, Feldman EL. Diabetic neuropathy: cellular mechanisms as therapeutic targets. Nat Rev Neurol 7: 573-583.

12. Chen PC Lao CL, Chen JC. The $\mathrm{D}(3)$ dopamine receptor inhibits dopamine release in PC-12/hD3 cells by autoreceptor signaling via PP-2B, CK1, and Cdk-5. J Neurochem 2009; 110: 1180-1190

13. Manschot SM, Biessels GJ, de Valk H, Algra A, Rutten GE, van der Grond J et al. Metabolic and vascular determinants of impaired cognitive performance and abnormalities on brain magnetic resonance imaging in patients with type 2 diabetes. Diabetologia 2007 50: 2388-2397.

14. Selvarajah D, Wilkinson ID, Davies J, Gandhi R, Tesfaye S. Central nervous system involvement in diabetic neuropathy. Curr Diab Rep 2011; 11: 310-322.

15. Melrose HL, Kinloch RA, Cox PJ, Field MJ, Collins D, Williams D. [3H] pregabalin binding is increased in ipsilateral dorsal horn following chronic constriction injury. Neurosci Lett 2007 417: 187-192.

16. Drolet RE, Sanders JM, Kern JT. Leucine-rich repeat kinase 2 (LRRK2) cellular biology: a review of recent advances in identifying physiological substrates and cellular functions. J Neurogenet 25: 140-151.

17. Gomez-Suaga P, Fdez E, Blanca Ramirez M, Hilfiker S. A link between autophagy and the pathophysiology of LRRK2 in Parkinson's disease. Parkinsons Dis 2012 2012: 324521.

18. Gomez-Suaga P, Luzon-Toro B, Churamani D, Zhang L, Bloor-Young D, Patel S et al. Leucine-rich repeat kinase 2 regulates autophagy through a calcium-dependent pathway involving NAADP. Hum Mol Genet 2012; 21: 511-525.

19. Drolet RE, Sanders JM, Kern JT. Leucine-rich repeat kinase 2 (LRRK2) cellular biology: a review of recent advances in identifying physiological substrates and cellular functions. J Neurogenet 2011; 25: 140-151.

20. Greggio E, Cookson MR. Leucine-rich repeat kinase 2 mutations and Parkinson's disease: three questions. ASN Neuro 2009; 1: e00002.

21. Shin YK, Jang SY, Lee HK, Jung J, Suh DJ, Seo SY et al. Pathological adaptive responses of Schwann cells to endoplasmic reticulum stress in bortezomib-induced peripheral neuropathy. Glia 2010; 58: 1961-1976.

22. Locke S. Axons, Schwann cells, and diabetic neuropathy. Bull NY Acad Med 1967; 43 784-791.

23. McCandless DW, Feussner GK, Lust WD, Passonneau JV. Sparing of metabolic stress in Purkinje cells after maximal electroshock. Proc Natl Acad Sci USA 1979; 76: 1482-1484.

24. MacKenzie-Graham A, Tiwari-Woodruff SK, Sharma G, Aguilar C, Vo KT, Strickland LV et al. Purkinje cell loss in experimental autoimmune encephalomyelitis. Neuroimage 2009; 48: 637-651.

25. Sudarov A. Defining the role of cerebellar purkinje cells in autism spectrum disorders Cerebellum 2013; 12: 950-955.

26. Kasumu A, Bezprozvanny I. Deranged calcium signaling in Purkinje cells and pathogenesis in spinocerebellar ataxia 2 (SCA2) and other ataxias. Cerebellum 2012; 11: 630-639.

27. Wang QJ, Ding Y, Kohtz DS, Mizushima N, Cristea IM, Rout MP et al. Induction of autophagy in axonal dystrophy and degeneration. J Neurosci 2006; 26: 8057-8068.

28. Komatsu M, Waguri S, Chiba T, Murata S, Iwata J, Tanida I et al. Loss of autophagy in the central nervous system causes neurodegeneration in mice. Nature 2006; 441: 880-884.

29. Jiang YQ, Wang XL, Cao XH, Ye ZY, Li L, Cai WQ. Increased heat shock transcription factor 1 in the cerebellum reverses the deficiency of Purkinje cells in Alzheimer's disease. Brain Res 2013; 1519: 105-111.

30. Rajput $\mathrm{AH}$, Robinson $\mathrm{CA}$, Rajput ML, Rajput A. Cerebellar Purkinje cell loss is not pathognomonic of essential tremor. Parkinsonism Relat Disord 2011; 17: 16-21.

31. Chan L, Terashima T, Urabe H, Lin F, Kojima H. Pathogenesis of diabetic neuropathy: bad to the bone. Ann NY Acad Sci 2011; 1240: 70-76.

32. Weimann JM, Johansson CB, Trejo A, Blau HM. Stable reprogrammed heterokaryons form spontaneously in Purkinje neurons after bone marrow transplant. Nat Cell Biol 2003; 5 959-966.
33. Wei M, Ong L, Smith MT, Ross FB, Schmid K, Hoey AJ et al. The streptozotocin-diabetic rat as a model of the chronic complications of human diabetes. Heart Lung Circ 2003; 12: 44-50.

34. Nishi A. [Role of Cdk5 in dopamine signaling and addiction]. Tanpakushitsu Kakusan Koso 2009; 54: 813-819.

35. Chakrabarti L, Eng J, Ivanov N, Garden GA, La Spada AR. Autophagy activation and enhanced mitophagy characterize the Purkinje cells of pcd mice prior to neuronal death. Mol Brain 2009; 2: 24.

36. Plowey ED, Cherra 3rd SJ, Liu YJ, Chu CT. Role of autophagy in G2019S-LRRK2associated neurite shortening in differentiated SH-SY5Y cells. J Neurochem 2008; 105 : 1048-1056.

37. Kawakami F, Yabata T, Ohta E, Maekawa T, Shimada N, Suzuki M et al. LRRK2 phosphorylates tubulin-associated tau but not the free molecule: LRRK2-mediated regulation of the tau-tubulin association and neurite outgrowth. PLoS One 7: e30834.

38. Wang X, Yan MH, Fujioka H, Liu J, Wilson-Delfosse A, Chen SG et al. LRRK2 regulates mitochondrial dynamics and function through direct interaction with DLP1. Hum Mol Genet 2012; 21: 1931-1944.

39. Yamano T, Shimada M, Fujizeki Y, Kawasaki H, Onaga A. Quantitative synaptic changes on Purkinje cell dendritic spines of rats born from streptozotocin-induced diabetic mothers. Brain Dev 1986; 8: 269-273.

40. Saha S, Liu-Yesucevitz L, Wolozin B. Regulation of Autophagy by LRRK2 in Caenorhabditis elegans. Neurodegener Dis 2014; 13: 110-113.

41. Avila J, Lucas JJ, Perez M, Hernandez F. Role of tau protein in both physiological and pathological conditions. Physiol Rev 2004; 84: 361-384.

42. Zhang Y, Tian Q, Zhang Q, Zhou X, Liu S, Wang JZ. Hyperphosphorylation of microtubuleassociated tau protein plays dual role in neurodegeneration and neuroprotection. Pathophysiol 2009; 16: 311-316.

43. Kageyama Y, Zhang Z, Roda R, Fukaya M, Wakabayashi J, Wakabayashi N et al. Mitochondrial division ensures the survival of postmitotic neurons by suppressing oxidative damage. J Cell Biol 197: 535-551.

44. Cameron NE. Role of endoplasmic reticulum stress in diabetic neuropathy. Diabetes 62 : 696-697.

45. Portha B, Picon L, Rosselin G. Chemical diabetes in the adult rat as the spontaneous evolution of neonatal diabetes. Diabetologia 1979; 17: 371-377.

46. Blondel $\mathrm{O}$, Bailbe $\mathrm{D}$, Portha B. Relation of insulin deficiency to impaired insulin action in NIDDM adult rats given streptozocin as neonates. Diabetes 1989; 38: 610-617.

47. Heffner TG, Hartman JA, Seiden LS. A rapid method for the regional dissection of the rat brain. Pharmacol Biochem Behav 1980; 13: 453-456.

48. Taymans JM, Van den Haute C, Baekelandt V. Distribution of PINK1 and LRRK2 in rat and mouse brain. J Neurochem 2006; 98: 951-961.

49. Harris KM, Stevens JK. Dendritic spines of rat cerebellar Purkinje cells: serial electron microscopy with reference to their biophysical characteristics. J Neurosci 1988; 8: $4455-4469$.

(c) (i) () $\odot$ Cell Death and Disease is an open-access journal published by Nature Publishing Group. This work is licensed under a Creative Commons Attribution-NonCommercialNoDerivs 3.0 Unported License. The images or other third party material in this article are included in the article's Creative Commons license, unless indicated otherwise in the credit line; if the material is not included under the Creative Commons license, users will need to obtain permission from the license holder to reproduce the material. To view a copy of this license, visit http://creativecommons.org/ licenses/by-nc-nd/3.0/ 